\title{
Surface Electromyography Signal Collecting Strategy for Artificial Hand Controlling Purposes
}

\author{
Abdul-Sahib N. Kadhim, Mohammed M. Abdul-Sattar, and Hasan S. Falah
}

\begin{abstract}
Surface electromyography technique is presented for muscle activation detection and intuitive control of prostheses or robot arm. This research aims to develop a strategy for collecting EMG signal in order to be used as input control for an artificial hand. Methodology of this research composed of, collecting EMG signal using clinical device to choose the best position. Collect the signal using myoware muscle sensor depending on these positions locating above with determining the threshold value for each position. The test was done on a healthy female person and an amputee person, a number of three position were used by the clinical myotrace 400 each positon composed of four channels $i$, four channel location were chosen from the three position that were used by the myoware sensor on the forearm in addition to two locations on the ulna, while the amputee included only two locations on the ulna. The locations obtained from the myotrace targeting muscles (extensor carpi ulnaris, flexor digitorium sublimis, brachioradialis, flexor carpi ulnaris). The threshold values obtained were on a range of 150-320 that consider a nonsensitive. For the amputee the threshold values were the form 150-220 for both the healthy arm and the amputee arm which consider a weaker value.
\end{abstract}

Index Terms-Surface Electromyography; Threshold Value; Electrodes Positions; Artificial Hand.

\section{INTRODUCTION}

has been demonstrated that the signal gathered from the muscle can be utilized to generate input commands for the control of prosthetic hand [1]. With the change of electromyography strategy, the surface EMG signal has turned into a progressive means for control of prosthetics upper limbs [1].

Electromyography signal is an aggregate electrical signal from skeletal muscle which denotes the electrical activity of a muscle during contraction. In the previous years there has been a colossal enthusiasm for the presenting natural interfaces that can perceive the user's body developments and convert into machine commands. EMG signal is random signal which is controlled by sensory system which is reliant on the anatomical and physiological properties of muscles. EMG signal acquires noise in very high degree which can be excluded by good implantation of electrodes and good wire connections of sensors. [2].

The RAW EMG signals consider a very fluctuated signals with a low voltage $(\sim 5$ microvolt $)$ that only gives a representation of the excitation of the muscle. Most clinical devises record this signal without giving further signals processing. These signal can't be used as input commands

Published on October 20, 2017

A. N. Kadhim, M. M. Abdul-Sattar, and H. S. Falah are with Mechanical Engineering Department, College of Engineering, Al Nahrain University, Iraq. (e-mail: n_k_alsahib@yahoo.com, m1976sjnr@gmail.com,samahflah@gmail.com) for a most controlling units, thus, in order to make it allowable to be used as commands the signal need to be filtered, magnified, and optimized these procedures are done by using special designed circuits that are designed for this propose, such circuits are myoware muscle sensor developed by the advancer technologies company.

MSC can be isolated essentially into two gatherings pattern recognition and non-pattern recognition based. In the primary gathering, the desired functions are segregated from signal patterns by classifiers and the assortment of capacities depends specifically on characterization execution. While the non-pattern recognition based controllers are constructed on threshold control and or potentially limited state machines only yield restricted and pre characterized control charges in light of an arrangement of info signal patterns [3].

The signal-threshold technique is an essential and basic phenomenological approach. In this technique, rectified raw EMG (Fig. 3-17) is compared with thresholds that are acquired based on the mean power of background noise. It is quick and straightforward in usage. It suits coarse ON/OFF location instead of its moment esteem.

Tremendous amount of works was done previously concerning with recording of EMG signal such as [4] as they introduced a framework that enabled an individual finger movement using sEMG data collected by 32 bipolar electrodes placed on the ble-bodied individual forearm while performing 10 separate finger movements using traditional signal analysis tool [5].

Studied the upper limb muscle activities during basic upper limb motion in daily works to help power assist robotic exoskeleton systems to estimate upper limb motions in based on electromyography signal collected from the related muscles and used as input to control the exoskeleton [6].

Represented the relationship between the thumb tip forces and electromyogram signal (EMG) to develop more natural controlled prosthetic thumb; They depend on the Raw EMG that are classified by the neural network based on different force and thumb configuration and collected from the thumb intrinsic muscles.

Myotrace 400 devise prove to be a very useful in the field of the recording and measurements of the muscle activation. For instant, [7] used the devise to measure the acceleration and velocity (vibrational data) of human arm of hard works. And also implement the device in measuring the vibrational data of older people with hip replacement.

In this work EMG signal generated from the excitation of the upper limb is recorded by two methods. Both clinical device and a controller sensor is used to locate the best position to generate the efficient signal that can be used as control input to an artificial hand. first the signal was recorded using a clinical device from four channel on the 
forearm in three sets, then the best positions were chosen to use them as efficient locations for the sensor that will be used as controller input. The generated signal was compared and tested on a five finger artificial hand moved by servo motors.

\section{METHODOLOGY}

The method summarized in two sections, first using the myo Trace 400 clinical device to record the EMG signal, then after locating the best positions, we implement the myoware muscle sensor and observe the generated signal with locating the threshold value that can be used as input value for controlling of artificial hand.

\section{A. Myotrace 400 signal recording}

The myotrace 400 consider the latest portable handheld measurement rechnology that is designed and manufactured by naraxon coopration for clinician interested accurate and quick measurements. It can be used for EMG evaluation and biofeedbach with sEMG sensors. It can be connected with four sEMG sensors which gives the opportunity of recording four muscle excitation in the same time. It can be connected to $\mathrm{PC}$ and used for more advance analyzing with the MR3(Muscle Research 3) software.

Steps of the test procedure

1- Locating the positions required.

2- Preparing the skin of the volunteer.

3- Apply the surface electrodes.

4- Connect the sensors

5- Connect the device to PC and open the software.

The test was done on a 17 years female person weigh $60 \mathrm{~kg}$ for three sets, each set represent a four positions of the four electrodes. The test protocol considered seven movements with a seven interval of rest. The movement which consider an excitation of the muscle, last for 5 second following a rest interval of 5 second as well and so on to the next movement. The movements involved are, in sequence, full hand close, full hand open, pinch, four fingers full close, index full close, ring finger full close, wrist flexion.

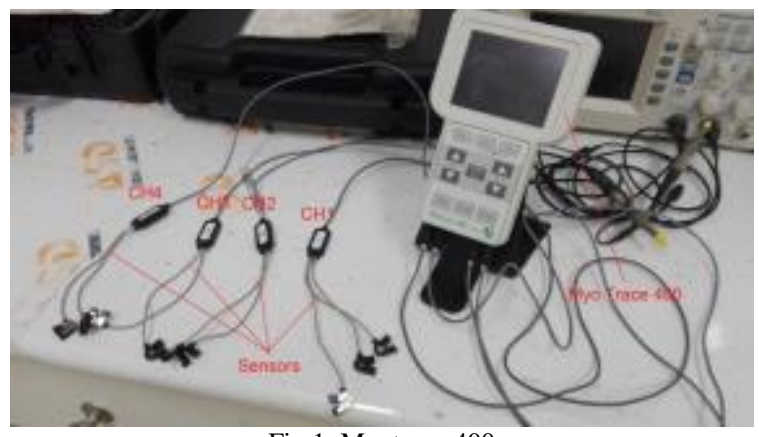

Fig.1. Myotrace 400

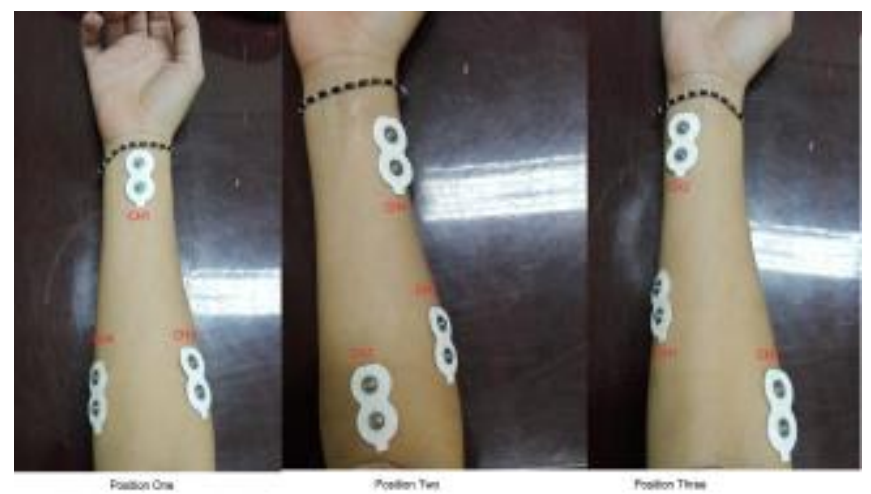

Fig.1. Channel location (interior view ).

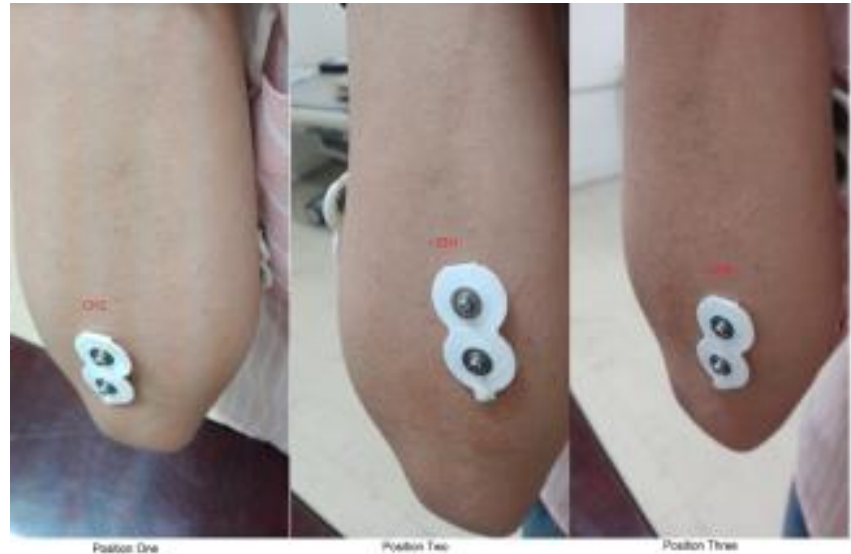

Fig.3. Channels locations (posterior view)
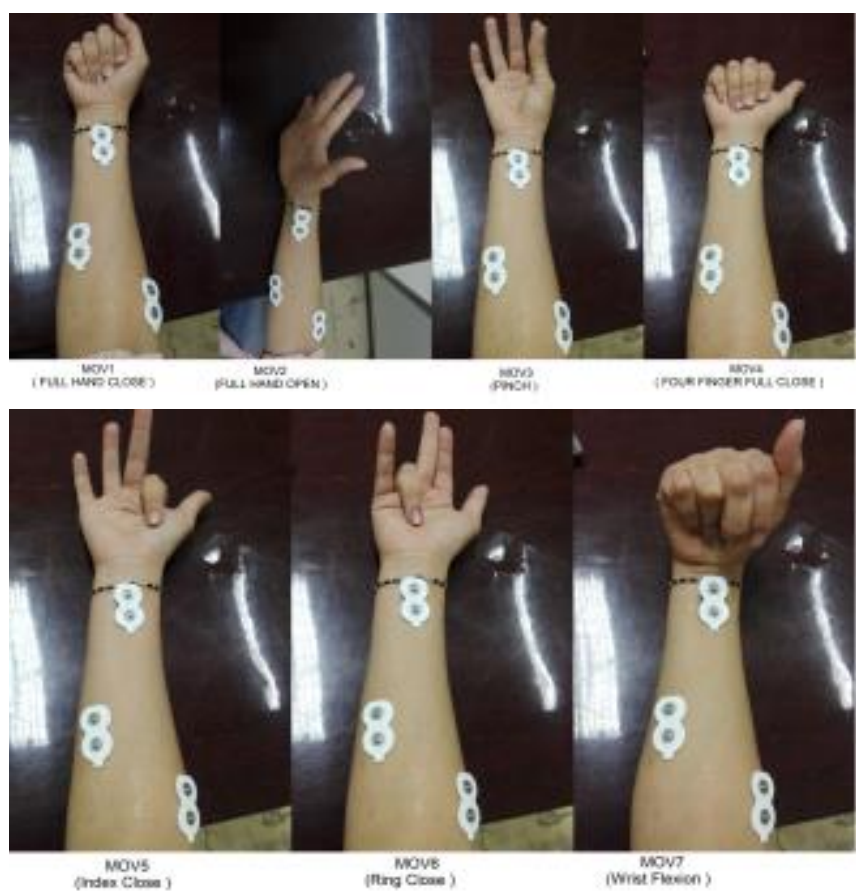

Fig.4. Movements required

\section{B. Myoware muscle sensor signal recording}

It is a 3 lead muscle sensor designed especially for collecting surface EMG signal. It is a very sophisticated electronic circuit that can be used in the field of robotic and prosthetic. It can be connected with any controller such as the Arduino mega 2560 which is used in this work. 


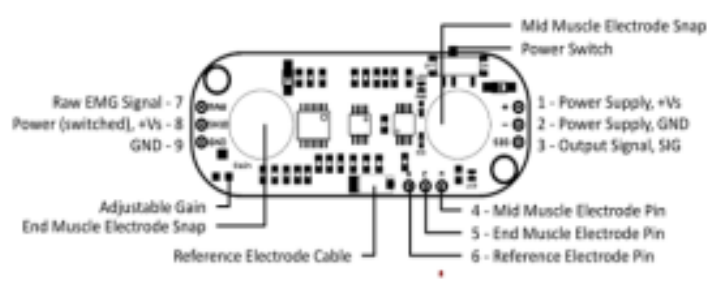

Fig.5. Schematics of myoware muscle sensor

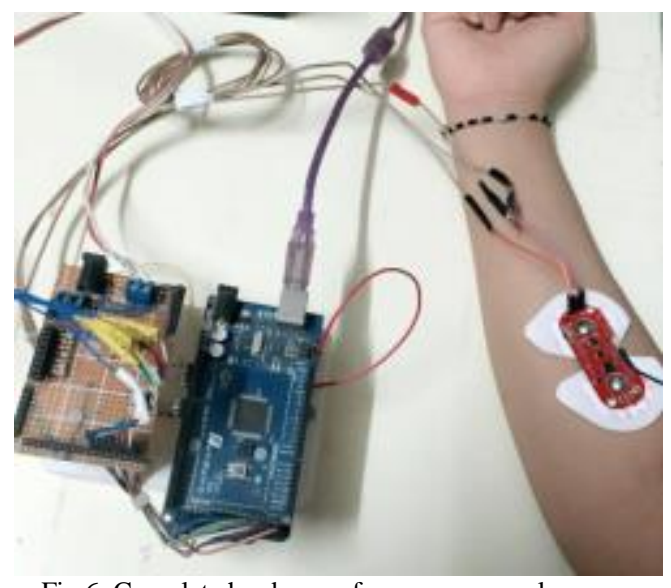

Fig.6. Complete hardware of myoware muscle sensor

The test involves four positions on the forearm also extra ones on the ulna were targeted for the healthy person. For the amputee person Only two positions were located, one on the biceps(X1) and the other in the middle radial distance between biceps and triceps (X2), the same positions were located on the healthy arm (Left arm) so we can make a comparison between the healthy and the amputated arm.
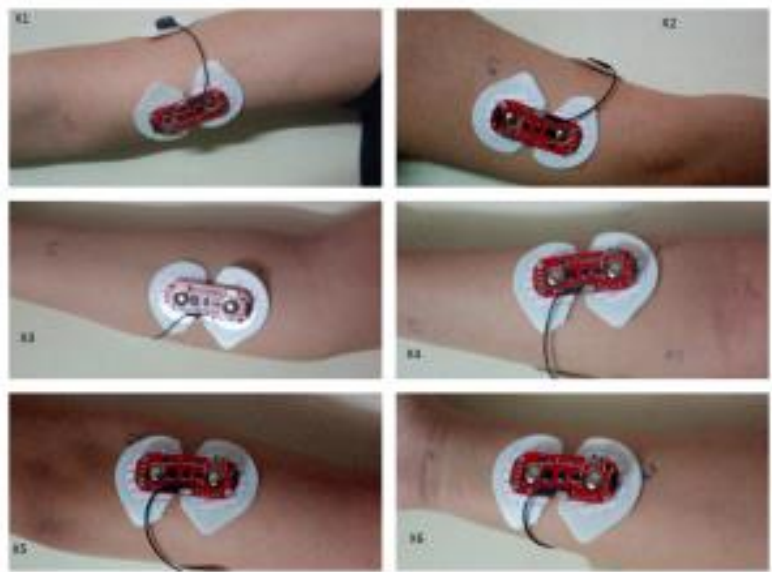

Fig.7. Myoware muscle sensor locations

TABLE I: POSITIONS WITH TARGETING MUSCLE

\begin{tabular}{ll} 
& TABLE I: POSITIONS WITH TARGETING MUSCLE \\
\hline \hline Position & Muscle \\
\hline X1 & Biceps \\
X2 & Triceps \\
X3 & Flexor Carpi Ulnaris \\
X4 & Brachioradialis \\
X5 & Extensor Carpi Ulnaris \\
X6 & Flexor D Digitorum \\
\hline \hline
\end{tabular}

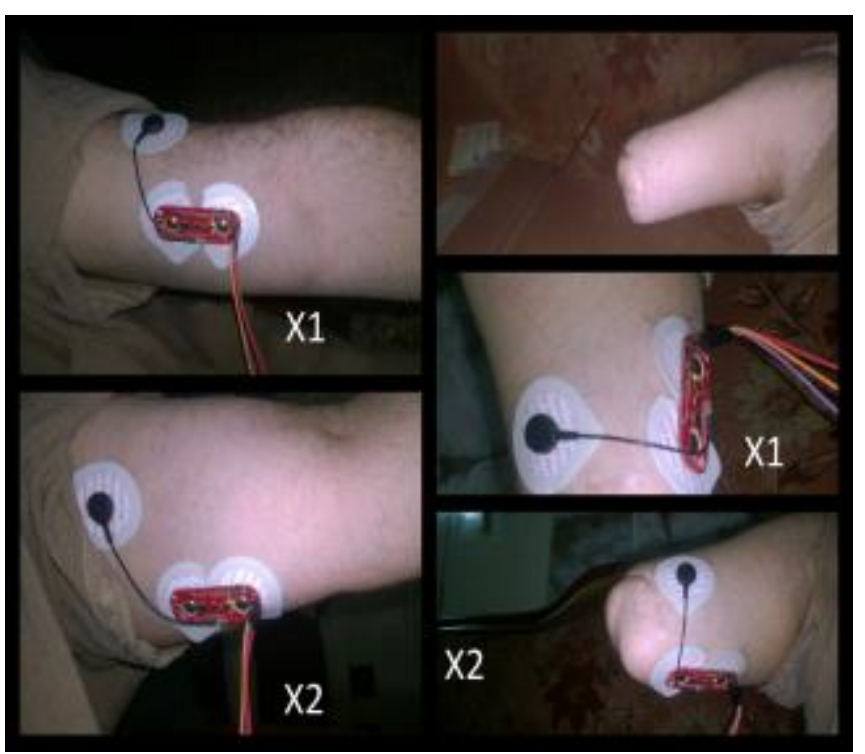

Fig.8. Myoware muscle sensot location on the amputee

\section{RESULT}

\section{A. Myotrace 400}

The MR3 software gives the possibility to examine the response of all four channels i.e muscle with a signal movement. Fig. 9 to 11 show the generated excitation of each channel with the sets of movements. Each row represents each channel.

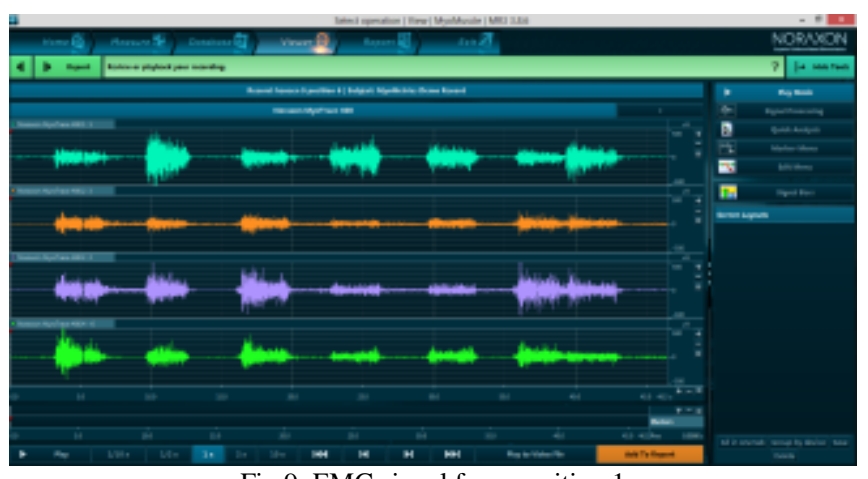

Fig.9. EMG signal from position 1

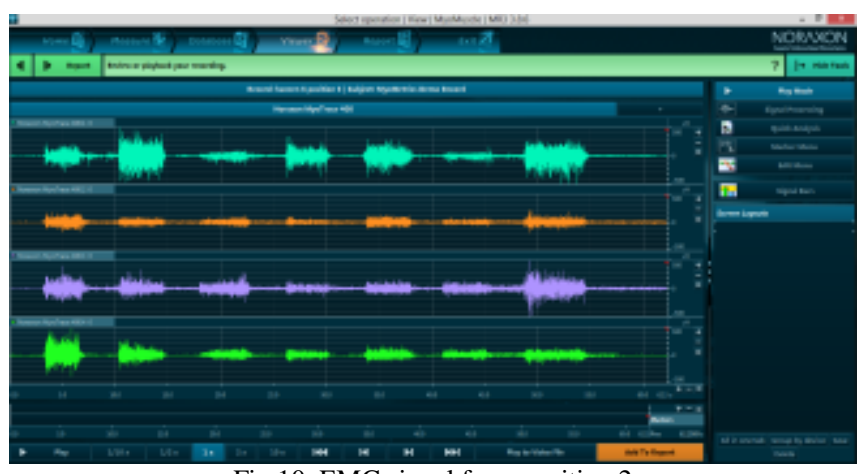

Fig.10. EMG signal from position 2 


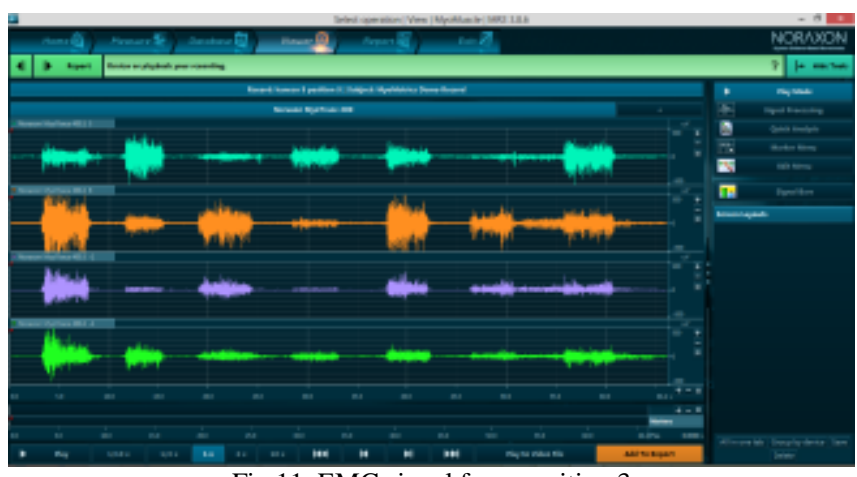

Fig.11. EMG signal from position 3

For position 1 the location of $\mathrm{CH} 1, \mathrm{CH} 2, \mathrm{CH} 3$ consider suitable positions for obtaining valuable EMG signal. For position $2 \mathrm{CH} 1, \mathrm{CH} 4$ and slightly $\mathrm{CH} 3$ were able to differentiate and record the signals for a number of movements. For position $3 \mathrm{CH} 2$ obviously represents the best suited position from this test. Finally, we can say that the best chosen locations from the three set are:

TABLE II: Best Channels and Positions

\begin{tabular}{lll}
\hline \hline Channel & Test & Muscle \\
\hline CH1 & Position Two & Extensor Carpi Ulnaris \\
$\mathrm{CH} 2$ & Position Three & Flexor Digitorum Sublimis \\
$\mathrm{CH} 3$ & Position One & Brachioradi-alis \\
$\mathrm{CH} 4$ & Position One & Flexor Carpi Ulnaris \\
\hline \hline
\end{tabular}

\section{B. Myoware muscle sensor}

\section{1) Healthy person}

After determining the best positions by the Myotrace, they were used in the myoware muscle sensor. we used a some extra positions on the ulna to give the observe the signal as with conserving with the amputee person After recording the signal, threshold value is set for each position.

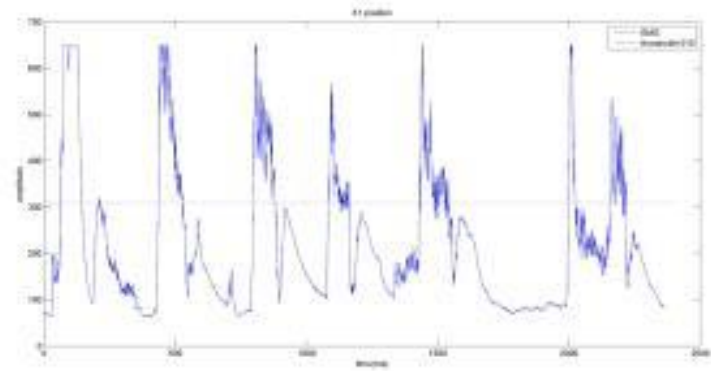

Fig.12. Generated EMG signal from X1

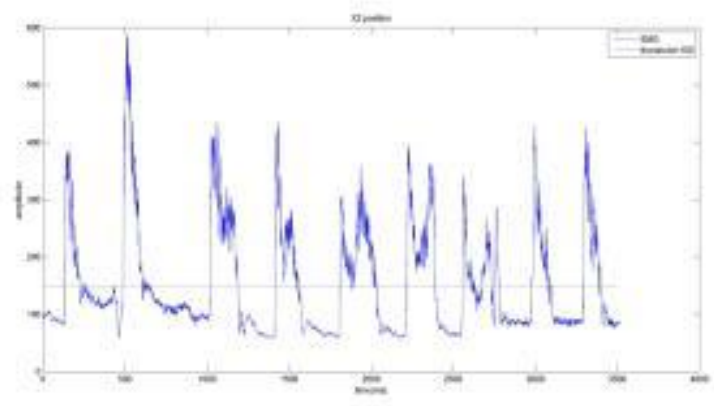

Fig.13. Generated EMG Signal From X2

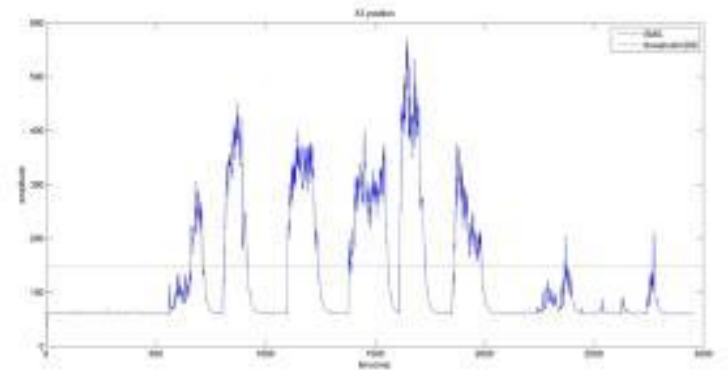

Fig.14. Generated EMG Signal from X3

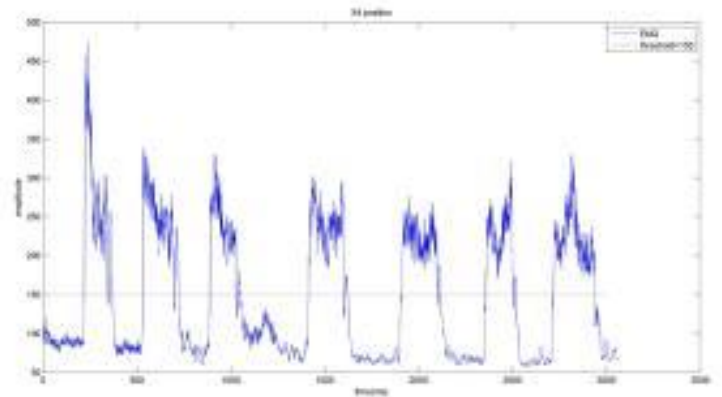

Fig.15. Generated EMG Signal from X4

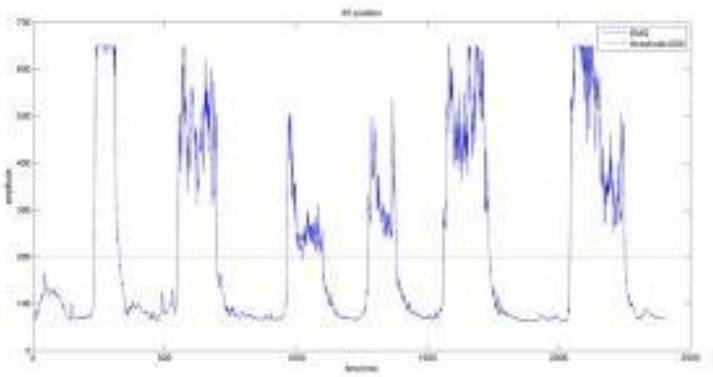

Fig.16. Generated EMG Signal from X5

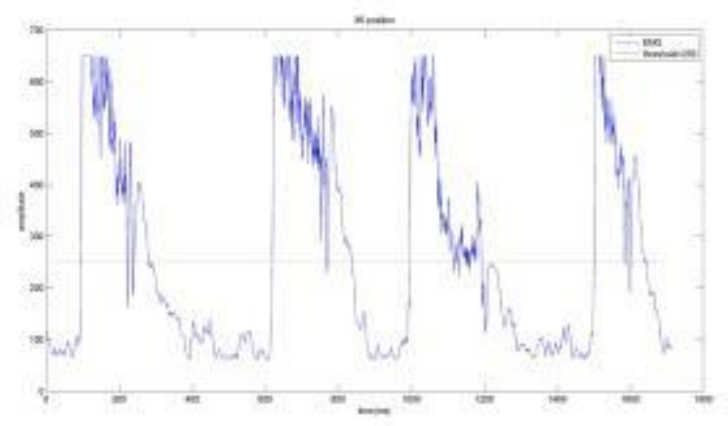

Fig.17. Generated EMG Signal from X6

2) Amputee person

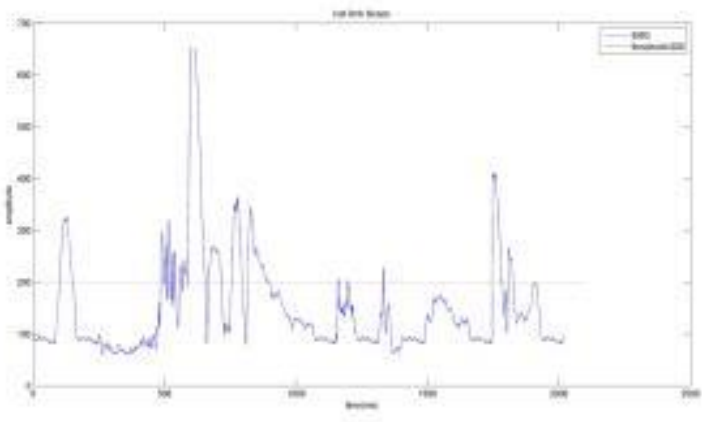

Fig.18. Generated EMG for the lost limb triceps 


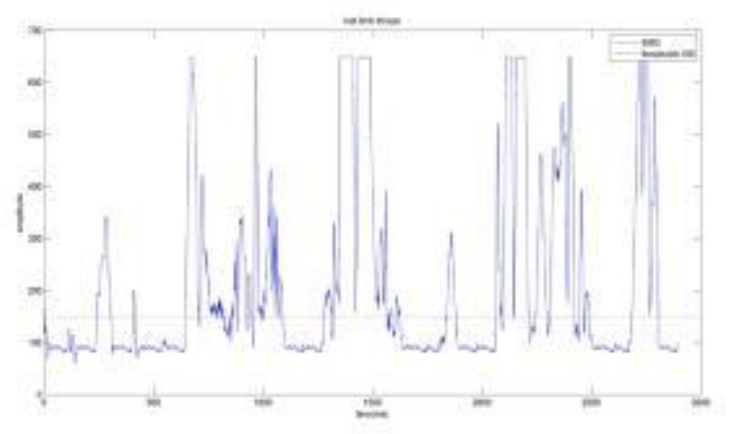

Fig.19. Generated EMG for the lost limb biceps

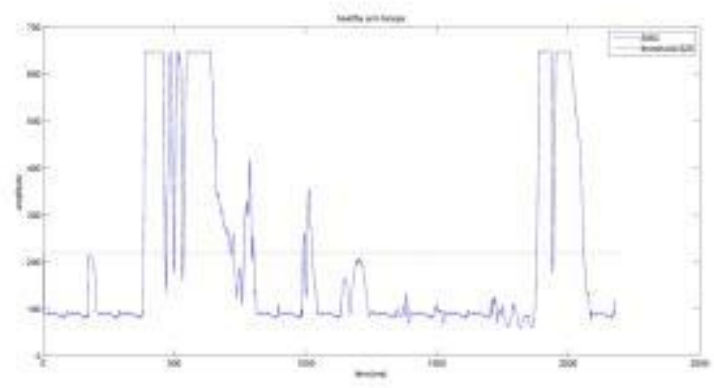

Fig.20. Generated EMG for healthy arm biceps

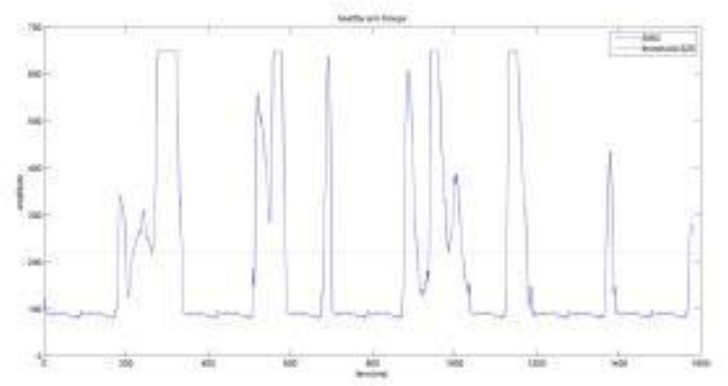

Fig.2. Generated EMG for healthy arm triceps

\section{Obtaining the threshold value}

1) Healthy Person

\begin{tabular}{ll}
\multicolumn{2}{c}{ TABLE III: Healthy Person } \\
\hline \hline Position & Threshold \\
\hline X1 & 310 \\
X2 & 150 \\
X3 & 200 \\
X4 & 150 \\
X5 & 200 \\
X6 & 250 \\
\hline \hline
\end{tabular}

\section{2) Amputee person}

TABLE IV: Amputee Person Lost Limb Threshold

\begin{tabular}{ll}
\hline \hline Position & Threshold \\
\hline X1(Biceps) & 200 \\
X2 (Triceps) & 150 \\
\hline \hline
\end{tabular}

TABLE V: Amputee Person Healthy Limb Threshold

\begin{tabular}{ll}
\hline \hline Position & Threshold \\
\hline X1(Biceps) & 220 \\
X2 (Triceps) & 220 \\
\hline \hline
\end{tabular}

\section{CONCLUSION}

The residual limb test showed a promising ability of working with the proposed design of the artificial hand. The EMG signal recorded is valuable that has a clear, located threshold value that can control the artificial hand. The degree of amputation of the limb affects highly the performance of the muscle, thus, affecting the ability to control the artificial hand. In order to control the artificial hand, it was noticed that the muscle needs to be strong without any stress. These factors affected highly the recorded EMG signal from muscles (amputee person, myo trace 400 test) which represent the muscle condition. There is no big difference of the EMG values of multiple muscles of individual, thus, a single value can be chosen for the number of muscle, i.e, location.

\section{REFERENCES}

[1] S. Guo, M. Pang, B. Gao, H. Hirata and H. Ishihara, "Comparison of sEMG-Based Feature Extraction and Motion Classification Methods for Upper-Limb Movement," sensors , pp. 9023-9037, 2015.

[2] M. . B. I. Reaz, M. . S. Hussain and F. Mohd-Yasin, "Techniques of EMG signal analysis: detection, processing, classification and applications," Malaysia , 2006.

[3] M. A. Oskoei and H. Hu, "Myoelectric control systems-A survey," Elesvier ,Biomedical Signal Processing and Control, pp. 276-289-290, 2007.

[4] F. Tenore, A. Ramos, A. Fahmy, S. Acharya, R. Etienne-Cummings and N. V. Thakor, "Towards the Control of Individual Fingers of a Prosthetic Hand Using Surface EMG Signals," in 29th Annual International Conference of the IEEE EMBS Cité Internationale, Lyon, France, 2007.

[5] R. A. R. C. Gopura, K. Kiguchi and E. Horikawa, "A Study on Human Upper-Limb Muscles Activities during Daily Upper-Limb Motions," International Journal of Bioelectromagnetism, vol. 12, no. 2,pp. $54-61,2010$

[6] S. N. Sidek, N. A. Jalaludin and A. U. Shamsudin, "Surface Electromyography (sEMG)-based Thumb-tip Angle and Force Estimation Using Artificial Neural Network for Prosthetic Thumb," Elesevier ,Procedia Engineering, pp. 650-656, 2012.

[7] J. S. Chiad, B. A. Bdawi and . A. J. Abbed, "Study the Effect of the Foundation Surface on the Vibration Data at the Worker Arm Using Drilling Machine," International Journal of Advanced Sport Sciences Research, pp. 539-547, 2015. 